\title{
Electronic-circuit-design principles for parameter control of i.s.f.e.t.s and related devices
}

\section{P. Bergveld}

Department of Electrical Engineering, Twente University of Technology, PO Box 217, Enschede, The Netherlands

\begin{abstract}
With the development of semiconductor transducers, such as i.s.f.e.t.s and related devices, a new class of transducers has been introduced in which the actual transducer function is integrated with part of the corresponding electronic circuitry. In the paper the transducer is therefore considered as being a new electronic component instead of being seen by the classical approach, in which the transducer and the electronic circuit are simply series connected. This view appears to result in unique possibilities for stabilisation and control of transducer characteristics. This is illustrated in the paper with respect to the effects of internal series resistances and the temperature sensitivity of is.f.e.t. devices.
\end{abstract}

Keywords-Electronic-circuit design, I.S.F.E.T.S, Parameter control

\section{Introduction}

BESIDES BEING used for electronic components, semiconductor materials were also introduced in the past for the fabrication of transducers. Temperature and light sensitive resistors and semiconductors strain gauges are well known examples of 1-port transducer devices. The application of these devices is usually realised by connecting them to a voltage or current source and to an amplifier.

Later on, transistor devices were also introduced as 2-port transducers in order to increase the sensitivity of the transducer. A phototransistor is an example of this development. More recently, the ionsensitive field-effect transistor (i.s.f.e.t.) has been added to this type of transducer (CHEUNG, 1978). Although for the application of conventional electronic components, like bipolar transistors and f.e.t.s, a broad field of electronic circuits is being designed in order to control and stabilise the function of these devices, it is striking that, up to now, this has hardly been the case for transistor transducers. The papers describing these transducers, such as, for instance, those concerning the i.s.f.e.t. and related devices, are more or less limited to considerations and discussions about the principal operation (BERGVELD et al., 1978) and the development of an adequate technology for fabrication (ESASHI et al., 1978), but do not deal with the specific possibilities of these devices in the sense of being electronic components. A possible reason for this phenomenon is that the conventional way of connecting a transducer to an electronic circuit is a simple one-way series connection. Several papers have dealt with the

\footnotetext{
First received 13th November 1978 and in final form 13th March 1979

$0140-0118 / 79 / 050655+07 \$ 01 \cdot 50 / 0$

(c) IFMBE: 1979
}

Medical \& Biological Engineering \& Computing integration of the actual semiconductor transducer and the peripheral electronic circuits, e.g. impedance transformers and signal processing circuits, and this possibility is being promoted as one of the specific advantages of semiconductor transducers. The principle of the series connection has, however, never been discarded up to now. In this way the development of i.s.f.e.t.s is also scarcely more than a combined potentiometric sensor in series with an m.o.s.f.e.t. amplifier, eventually with integrated circuitry for supply stabilisation and buffering.

With the aforementioned 'series approach', the problems with respect to transducer function control and stabilisation of device characteristics have to be fully solved by the solid-state technologist. This paper will, however, deal with some electronic concepts which can be used for parameter control of solid-state semiconductor transducers. In practice there will always be a discussion about whether a solution will be found in the implementation of adequate solid-state technology or in the development of new electronic concepts. This is, however, also the case with the application of more conventional semiconductor devices, and will often give rise to some controversial points of view. Either the technologist produces devices with guaranteed characteristics, or the electronic engineer accepts deviations, within certain limits, and develops his circuits in such a way that a stable function of the device can still be guaranteed.

The possibility of an electronic solution to meet various design and application problems with i.s.f.e.t. devices will be discussed in this paper with respect to the influence of the i.s.f.e.t. geometry on the device characteristics and the temperature dependence of its sensitivity and offset voltage. The principles described can also be used for other transistor transducers. 
2 Influence of the i.s.f.e.t. geometry on device characteristics

One of the advantages of using silicon as a base for semiconductor transducers is said to be the knowledge of silicon technology, which in principle makes it very easy to convert a new idea into an operating device in a relatively short time. This is completely true, but as soon as conceptual differences in devices occur, one must choose whether a new technology has to be developed or whether a less adequate conventional technology should be accepted. This latter solution can be chosen when it is possible to find an electronic concept that can meet the omissions in the technology.

Focusing our attention on the difference between an m.o.s.f.e.t. and an i.s.f.e.t., besides the change in gate material from metal to electrolyte, the method of contacting the actual source and drain is also necessarily quite different. With an m.o.s.f.e.t. the source and drain regions can, in principle, be completely evaporated with aluminium, which makes a very low resistance contact after alloying. On the contrary, with i.s.f.e.t.s this contact method is impossible because the gate has to be contacted by an electrolyte, which means that no metal contacts can exist in the vicinity of this area. Depending on the necessary length of the insulating lacquers over the oxidised source and drain region, the contact places are usually some millimetres away from the

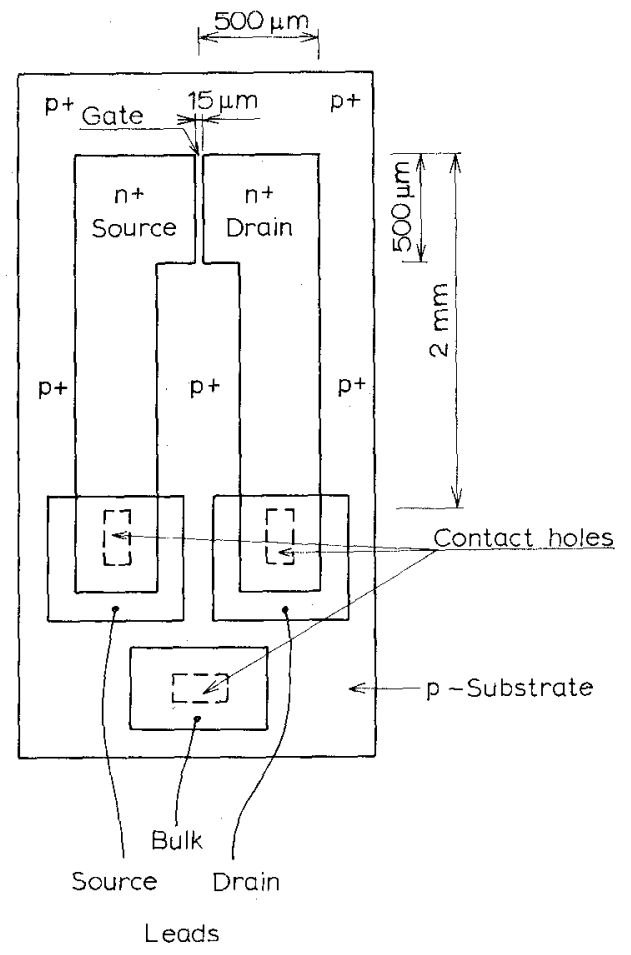

Fig. 1 Geometric representation of a common is.f.e.t. configuration actual source and drain-to-gate interface. The most common i.s.f.e.t. configuration is shown in Fig. 1 .

Using a donor concentration $N_{D}=5 \times 10^{19} \mathrm{~cm}^{-3}$ for the source and drain regions, resulting in a square resistance $R \square=40 \Omega$, internal source and drain resistances are created with a value $40 \times l / w \Omega$, in which $l / w$ is the length/width ratio of the diffusion region. In the case of the geometry as shown in Fig. 1, the series resistance of source and drain will thus be $4 \times 40 \Omega=160 \Omega$. In practical cases of needle-shaped i.s.f.e.t.s this value is even larger (ESASHI et al., 1978). This internal resistance of source and drain, of which the value depends, of course, on the actual geometry of the device, will never be zero, and gives rise to serious problems with respect to the sensitivity of the device. This can easily be seen if we consider the simple 1st-order equation for the drain current $I_{d}$ and the mutual conductance $S_{g}$ of an m.o.s.f.e.t.

$$
\begin{aligned}
& I_{d}=\beta\left[V_{d}\left(V_{\mathrm{g}}-V_{T}\right)-\frac{1}{2} V_{d}^{2}\right] \\
& S_{\mathrm{g}}=\left.\frac{d I_{d}}{d V_{\mathrm{g}}}\right|_{V_{d}=\text { const }}=\beta V_{d} \quad . \quad . \quad . \quad . \quad .
\end{aligned}
$$

in which $\beta$ is the geometry factor and $V_{T}$ the threshold voltage. In the case of the source and drain having internal resistance $R_{s}$ and $R_{d}$, respectively, the mutual conductance, or in other words the sensitivity of the device, will decrease to the effective mutual conductance

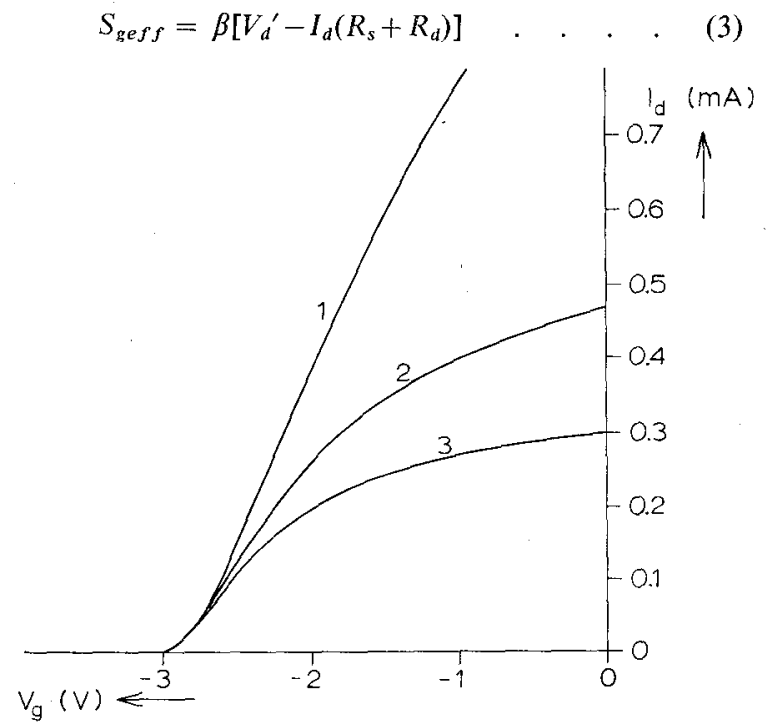

Fig. $2 I_{d} / V_{g}$ characteristics of a normal m.o.s.f.e.t. for various values of series resistors in source and drain leads $R_{s}$ and $R_{d r}$ respectively

a M.O.S.T. without $R_{s}$ or $R_{d}$

b M.O.S.T. with $R_{s}=120 \Omega$ or $R_{d}=120 \Omega$

c M.O.S.T. with $R_{s}=R_{d}=120 \Omega$

$V_{d s}=100 \mathrm{mV}$

$\beta=4 \times 10^{-3} \mathrm{AV}^{-2}$ 
where $V_{d}^{\prime}$ is the external applied drain to source voltage. This means that the sensitivity decreases as a function of $\left(R_{s}+R_{d}\right)$ and $I_{d}$, resulting in a bending of the $I_{d}-V_{g}$ curves, and even more for smaller values of $V_{d}{ }^{\prime}$. This effect is shown in the curves of Fig. 2, measured with a normal m.o.s.t. without and with additional $R_{s}$ and $R_{d}$.

The shape of the $I_{d} / V_{\mathrm{g}}$ curves measured with i.s.f.e.t.s can be fully explained as being the result of the internal source and drain resistances (EsASHI et al., 1978). Because only small values of $V_{d}$ can be used for i.s.f.e.t.s, the described effect is very pronounced (see eqn. 3 ) and will cause serious problems by the application of i.s.f.e.t.s.

In view of the considerations mentioned in Section 1, there are, in principle, two ways of solving this problem; namely, technologically and electronically. A technological way of approaching the problem is to contact the source and drain from the liquid-free side of the device. However, this implies new technologies because this technique is very unusual for standard transistor devices. The most promising solution is described by CLINE et al. (1976), while ZEMEL (1977) suggests that ic could be used for i.s.f.e.t.s and related devices. It is much easier to solve the problem of decreased sensitivity owing to internal source and drain series resistors by means of an electronic circuit, which is insensitive to series resistors due to the application of the feedback principle. The simplest way is to use a source follower system obtained by applying a current source in the source lead of the i.s.f.e.t. Also, the use of the bulk of the i.s.f.e.t. as an additional input lead for maintaining a feedback loop is reported (BERGVELD, 1972).
A more effective approach is, however, the following design of a forced source and drain follower with a possible additional bulk feedback, depending on eventual special requirements.

As can be seen in Fig. 3, the system consists essentially of a power supply (current source and adjustable reference voltage $V_{\text {ref }}$ ), an instrumentation amplifier system and an operational amplifier. The i.s.f.e.t. is connected to the leads of the instrumentation amplifier that are normally used to connect a resistor that determines the amplification of the amplifier. The usual inputs of the amplifier are, in this case, connected to a fixed voltage $I R_{1}$, provided by the current source. The output voltage of the instrumentation amplifier is now inversely proportional to the channel resistance of the i.s.f.e.t. Note that the connections for source and drain are of very low resistance, as in earlier designs, which means that the i.s.f.e.t. can be connected to the system by means of long unshielded wires (BERGVELD, 1968, 1972).

The difference between the output voltage of the instrumentation amplifier and an adjustable reference voltage $V_{r e f}$ is amplified by the operational amplifier, from which the output 'injects' a feedback current $I_{f}$ into $R_{2}$, resulting in a final source and drain voltage equal to $V_{R 2}$ and $V_{(R 2+R 1)}$, respectively. The current is measured via the adjustable resistor $R_{9}$. If the open-loop amplification of the system determined by the amplification of the combination of i.s.f.e.t. and instrumentation amplifier (approximately $R 8 / R 7 \times S_{\text {geff }} \times\left(R_{3}+R_{4}\right)$, and the open-loop amplification of the operational amplifier, is high enough, the source and drain potential with respect

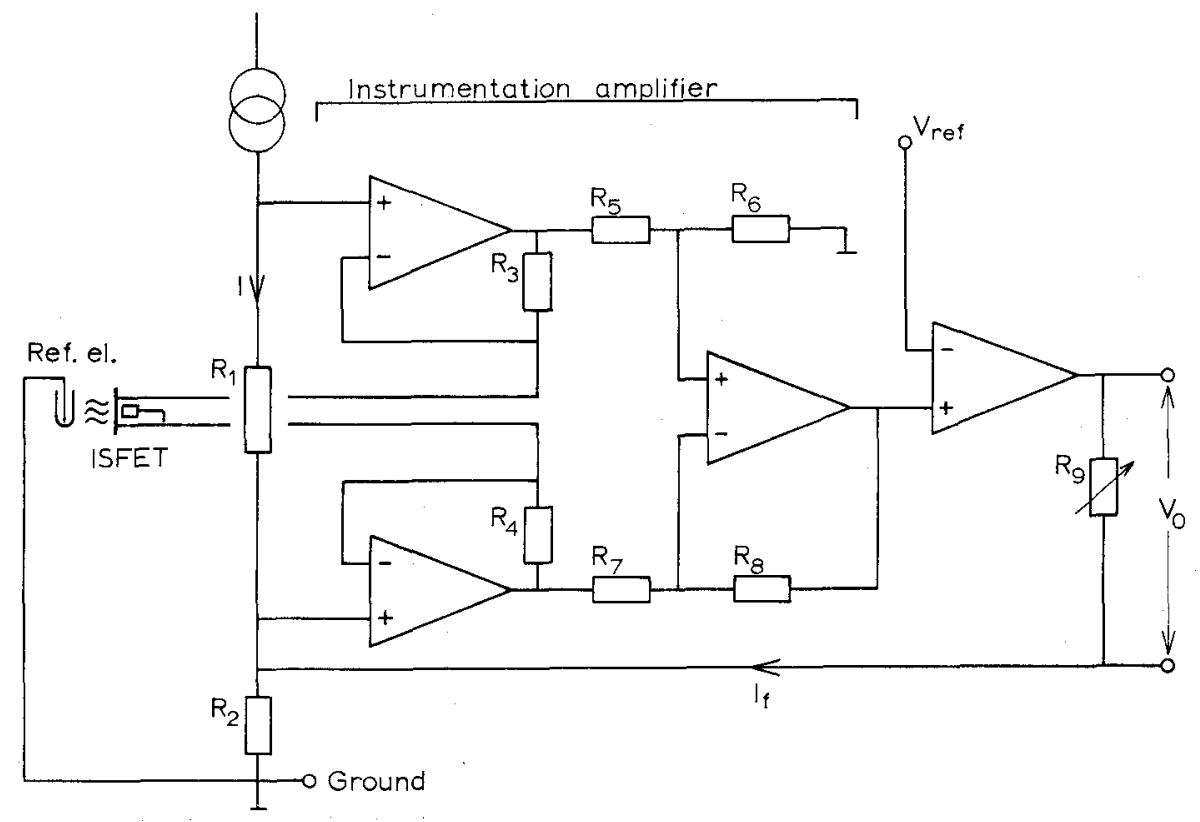

Fig. 3 Principle diagram of i.s.f.e.t. amplifier 
to earth follow a change in the input voltage $v_{i}$ of the i.s.f.e.t., resulting in an output voltage

$$
v_{0}=\frac{R_{9}}{R_{2}} v_{i}
$$

If we focus our attention on a $\mathrm{pH}$ sensitive i.s.f.e.t., the procedure of a $\mathrm{pH}$ measurement with this system is as follows. If the i.s.f.e.t. is placed in a buffer $\mathrm{pH}=7$, the reference voltage is adjusted in such a way that $I_{f}=0$. The output voltage across $R_{9}$ is thus also zero, independent of the value of $R_{9}$. If the i.s.f.e.t. is then placed in another buffer, e.g. $\mathrm{pH}=4$, the value of $R_{9}$ can be adjusted in such a way that an appropriate voltage is measured, e.g. $3 \mathrm{~V}$ if the desired sensitivity of the system should be $1 \mathrm{~V} / \mathrm{pH}$. Of course the output voltage can be directly calibrated in $\mathrm{pH}$ units.

Because the feedback of the system as shown in Fig. 3 is only effective if the liquid gate of the i.s.f.e.t. has a defined potential with respect to earth, the amplifier will not be biased as long as the i.s.f.e.t is not dipped in the liquid. Therefore, it is useful to connect the bulk not to the source, as commonly in use and shown in Fig. 3, but to the earth lead of the system. Then an additional bulk feedback loop is created by which the system will also be biased with an open gate (BERGVELD, 1972). Dipping the i.s.f.e.t. in the liquid now gives two feedback loops that are simultaneously active but do not influence the principal operation of the whole amplification system. Furthermore, the bulk itself can now be used as an earthed reference electrode if it is provided with a layer of $\mathrm{Ag} / \mathrm{AgCl}$. Summarising this Section, it has been shown that the sensitivity problems of i.s.f.e.t.s owing to internal source and drain resistances can be completely met by the design of an adequate electronic system.

\section{Temperature dependence of sensitivity and offset voltage of an i.s.f.e.t.}

A serious drawback of the application of semiconductor transducers is the temperature sensitivity of the devices. This implies that they are a bad proposition compared with other more conventional transducers. If we focus our attention on a $\mathrm{pH}$ measurement, it should be noticed that the usual $\mathrm{pH}$ glass electrode is, of course, also temperature dependent, but to solve this problem the $\mathrm{pH}$ equipment is provided with possibilities for compensation by means of control of the asymmetry potential and temperature correction for the slope, which is a commonly accepted procedure for the calibration of $\mathrm{pH}$ electrodes. With the application of a $\mathrm{pH}$ sensitive i.s.f.e.t., the temperature-dependent semiconductor characteristics of the probe give rise to additional temperature dependency of the measurement. If these additional effects of temperature dependence can be cancelled, the i.s.f.e.t. application can compete with the normal $\mathrm{pH}$ electrode, and the advantages of miniaturisation, solid-state character, rigidity and multiprobe possibilities can be then fully utilised.

Because the temperature-dependent semiconductor characteristics of an i.s.f.e.t. are essentially the same as those of an m.o.s.f.e.t., we will further consider in this Section the temperature dependency of m.o.s.f.e.t.s and discuss the possibilities of compensation for this temperature dependency. The following equation for the drain current describes all the effects that influence it (BERGVELD, 1969):

$$
\begin{aligned}
& I_{d}=\beta\left[\left(V_{\mathrm{g}}-V_{F B}-\phi_{B}\right) V_{d}-\frac{1}{2} V_{d}^{2}-\frac{2}{3}\right. \\
& \times\left.\times\left\{\left(V_{d}-V_{b}+\phi_{B}\right)^{\frac{3}{2}}-\left(-V_{b}+\phi_{B}\right)^{\frac{3}{2}}\right\}\right]
\end{aligned}
$$

in which $\beta$ is equal to $\mu_{n} C_{o x} w / l, \mu_{n}$ is the electron mobility in the inversion layer, $C_{o x}$ is the oxide capacitance per square area, $w / l$ is the geometry factor of the channel, $V_{\mathrm{g}}$ is the applied gate-source voltage, $V_{F B}$ is the flat band voltage, and

$$
\begin{aligned}
\phi_{B} & \left.=2 \frac{k T}{q} \ln \frac{N_{A}}{n_{l}} \text { (see eqn. } 7\right) \\
V_{d} & =\text { applied drain-source voltage } \\
\alpha & \left.=\left(2 \varepsilon_{0} \varepsilon_{S i} q N_{A}\right)^{\frac{1}{2}} / C_{o x} \text { (see eqn. } 7\right) \\
V_{b} & =\text { applied bulk-source voltage }
\end{aligned}
$$

In this equation, $\beta$ and $\phi_{B}$ are the temperaturedependent factors, resulting in an offset current $\Delta I_{d}=f(T)$ and a sensitivity $S_{g}=\beta V_{d}=g(T)$ (see eqn. 2). The sensitivity is thus temperature dependent, due to the parameter $\beta$, but, referring to Section 2, this influence can be cancelled by using the feedback system of Fig. 3, where the output/input relationship was insensitive to device parameters such as $\beta$. The resulting problem to be solved is the temperature-dependent offset current, which will be converted into a temperature-dependent offset voltage in the feedback system of Fig. 3.

In the literature, the temperature sensitivity of $\beta$ and $\phi_{B}$ is described as follows. The temperature sensitivity of $\beta=\mu_{n} C_{o x} w / l$ is due to the temperature sensitivity of the electron mobility in the inversion layer, given by the following equation:

$$
\frac{d \mu_{n}}{d T}=-m \frac{\mu_{n}}{T} \text {. . . . . . . . }
$$

in which LeISTIKo et al. (1965) found experimentally that $m=1.5$ for $-200^{\circ} \mathrm{C}<T<+200^{\circ} \mathrm{C}$, while VADASZ et al. (1966) found $m=1$ for $-55^{\circ}<$ $T<+125^{\circ} \mathrm{C}$. Therefore, for room temperature measurements, the latter case results in

$$
\frac{d \beta}{d T}=-\frac{\beta}{T} \quad . \quad . \quad . \quad . \quad . \quad . \quad . \quad .
$$


The temperature sensitivity for

$$
\phi_{B}=2 \frac{k T}{q} \ln \frac{N_{A}}{n_{i}}
$$

is also only empirically found, because the equation for the intrinsic carrier concentration

$$
n_{l}=C T^{-\frac{3}{2}} \exp \left(-\frac{W_{g S i}}{k T}\right)
$$

is an empirical relationship ( $C$ is an empiric constant, $W_{g S i}$ is the bandgap of silicon). From these equations it follows that:

$$
\begin{aligned}
\frac{d \phi_{B}}{d T}= & \frac{1}{T}\left[-\frac{3 k T}{q}+\phi_{B}-\frac{W_{\mathrm{g} S t}}{q}\right] \\
& \simeq \frac{1}{T}\left(\phi_{B}-\frac{W_{\mathrm{g} S i}}{q}\right) .
\end{aligned}
$$

Although the relationships for $d \beta / d T$ and $d \phi_{B} / d T$ are both confirmed by measurements, it will be obvious that it is no use substituting the relationship $\beta(T)$ and $\phi_{B}(T)$ in the equation for the drain current, hoping to find a relationship in which one temperature-dependent factor will clearly show up that could then be influenced by technological methods to achieve a simple, e.g. a linear, temperature dependency. Moreover, in practice the temperature dependency of $R_{s}$ and $R_{d}$, as described in Section 2, has also to be considered, which provides another complication.

If, however, $\Delta I_{d}(T)$ can be measured separately and simultaneously with $\Delta I_{d}\left(T, V_{g}\right)$, then a possibility exists to design an electronic system in which the influence of the temperature can be compensated. This means that without knowing the exact relation $\Delta I_{d}(T)$ concealed in $\Delta I_{d}\left(T, V_{g}\right)$, the signal $\Delta I_{d}(T)$ has to be withdrawn from the m.o.s.t. (or i.s.f.e.t.) itself. A measurement of $T$ with a separate sensor is not suitable, not even if this sensor is a second m.o.s.t. of a differential pair. The only solution is to find a measurable m.o.s.f.e.t. parameter, which is a function of $T$ in the same way as $I_{d}$ and which is not a function of the input voltage $V_{g}$. For this parameter the mutual conductance of the bulk $S_{b}$ is obviously an excellent candidate. Let us, therefore, consider the two equations for $I_{d}$ and $S_{b}$ :

$$
\begin{aligned}
I_{d}= & \beta\left[\left(V_{g}-V_{F B}-\phi_{B}\right) V_{d}-\frac{1}{2} V_{d}^{2}-\frac{2}{3}\right. \\
& \left.\times \alpha\left\{\left(V_{d}-V_{b}+\phi_{B}\right)^{\frac{3}{2}}-\left(-V_{b}+\phi_{B}\right)^{\frac{3}{2}}\right\}\right] \\
& \begin{aligned}
S_{b}= & \left.\frac{d I_{d}}{d V_{b}}\right|_{V_{d=\text { const }}} \\
= & \alpha \beta\left[\left(V_{d}-V_{b}+\phi_{B}\right)^{\frac{1}{2}}-\left(-V_{b}+\phi_{B}\right)^{\frac{1}{2}}\right] . . .
\end{aligned}
\end{aligned}
$$

in which the symbols have the usual meaning (see eqn. 4).

Under the condition that $V_{b}=0$ and with the approximation that $(1+x)^{m}=1+m x$, eqns. 8 and 9 become

$$
\begin{aligned}
& I_{d}=\beta\left[\left(V_{\mathrm{g}}-V_{F B}-\phi_{B}\right) V_{d}-\frac{1}{2} V_{d}^{2}-\alpha V_{d}\left(\phi_{B}\right)^{\frac{1}{t}}\right] \\
& S_{b}=\alpha \beta \frac{1}{2} V_{d} \phi_{B}^{-\frac{1}{2}} . . . . . . .
\end{aligned} .
$$

Assuming that due to a change in the temperature, $\beta$ and $\phi_{B}$ change according to

$$
\begin{aligned}
& \beta=\beta_{0}+\Delta \beta \text {. . . . . . . . . (12) } \\
& \phi_{B}=\phi_{B 0}+\Delta \phi_{B} \text {. }
\end{aligned}
$$

$I_{d}$ and $S_{b}$ will change correspondingly to

$$
\begin{aligned}
& I_{d}=I_{d 0}+\Delta I_{d} \quad \text {. . . . . . . . . } \\
& S_{b}=S_{b 0}+\Delta S_{b}
\end{aligned}
$$

$\beta_{0}, \phi_{B 0}, I_{d 0}$ and $S_{b 0}$ refer to the values at a certain initial temperature, thus being constants. Substitution of eqns. 12, 13, 14 and 15 in eqns. 10 and 11 and again with the approximation as mentioned above, it follows that:

$$
\Delta I_{d}=\frac{\Delta \beta}{\beta_{0}} I_{d 0}-\beta_{0}\left(1+\frac{\Delta \beta}{\beta_{0}}\right) V_{d} \Delta \phi_{B}\left(1+\frac{1}{2} \alpha \phi_{B 0}{ }^{-\frac{1}{2}}\right)
$$

$$
\Delta S_{b}=\frac{\Delta \beta}{\beta_{0}} S_{b 0}-\beta_{0}\left(1+\frac{\Delta \beta}{\beta_{0}}\right) V_{d} \Delta \phi_{B} \frac{1}{4} \alpha \phi_{B 0}-\frac{1}{2}
$$

in which $I_{d 0}$ is given by eqn. 10 with the subscript 0 in $S_{b 0}$ in the same way by eqn. 11 . If we are able to measure $\Delta S_{b}$, and, as we will see at the end of this Section, this can very easily be done, we have in fact also measured $\Delta I_{d}$ if $\Delta I_{d} / \Delta S_{b}$ is a constant. This relationship therefore has to be considered further.

$$
\frac{\Delta I_{d}}{\Delta S_{b}}=\frac{I_{d 0}+\eta F_{1}}{S_{b 0}+\eta F_{2}} . . \quad . \quad . \quad . \quad .
$$

in which

$$
\begin{aligned}
& \eta=\beta_{0}\left(\frac{\beta_{0}}{\Delta \beta}+1\right) V_{d} \Delta \phi_{B} \\
& F_{1}=\left(1+\frac{1}{2} \alpha \phi_{B 0}{ }^{-\frac{1}{2}}\right) \text {. } \\
& F_{2}=\frac{1}{4} \alpha \phi_{B 0}{ }^{-\frac{3}{2}}
\end{aligned}
$$

Depending on the values of the different parameters, three cases of operation of the device can be distinguished:
(a) $I_{d 0}>\eta F_{1}$ and $S_{b 0}>\eta F_{2}$
In this case $\Delta I_{d} / \Delta S_{b}=I_{d 0} / S_{b 0}=$ constant
(b) $\eta F_{1}>I_{d 0}$ and $\eta F_{2}>S_{b 0}$ 
In this case $\Delta I_{d} / \Delta S_{b}=F_{1} / F_{2}=$ constant because $\alpha$ and $\phi_{B 0}$ are constant

(c) In the intermediate range, if we denote $I_{d 0} / S_{b 0}=F_{3}$, it follows from eqn. 18 that

$$
\frac{\Delta I_{d}}{\Delta S_{b}}=F_{3} \frac{S_{b 0}-\eta F_{1} / F_{3}}{S_{b 0}-\eta F_{2}}
$$

This relation is only constant if $F_{1} / F_{2}=F_{3}$ or

$$
\frac{1+\frac{1}{2} \alpha \phi_{B 0} 0^{-\frac{1}{2}}}{\frac{1}{4} \alpha \phi_{B 0}{ }^{-\frac{3}{2}}}=\frac{I_{d 0}}{S_{b 0}}
$$

This equality can be controlled by the proper adjustment of $I_{d 0}$, which is an independent variable. The conclusion is that a separately measured signal, which is directly related to $\Delta S_{b}$, multiplied by a constant factor, or, in other words, properly amplified, gives a signal $\Delta I_{d}(T)$ that is independent of $V_{\mathrm{g}}$ but in the same way dependent on $T$ as $I_{d}\left(V_{g}, T\right)$.

The realisation of the described principle is quite simple. In order to create a sensitivity independent of $\beta$, the feedback system of Fig. 3 is used. In this system an additional sinusoidal signal is injected by means of a transformer that is connected between the source and the bulk. This is shown in Fig. 4.

Because the whole feedback system is limited to a frequency of $3 \mathrm{kHz}$, the frequency of the bulk signal is chosen as $30 \mathrm{kHz}$, and is thus not affected by the feedback loop. The amplitude of the $30 \mathrm{kHz}$ signal is measured at the output of the instrumentation amplifier by means of a lock-in amplifier and appears to be proportional to $\Delta S_{b}$. Of course, in this system the actual output of the system is not $I_{d}$ but $V_{0}$, which is, however, essentially the same. It appears that the curves $V_{0}(T)$ and $V_{s b}(T)$ are of the same shape, which can best be illustrated by a simultaneous recording of these signals, as shown in Fig. 5, where the m.o.s.f.e.t. under investigation was warmed up by a hair drier.

In addition, a negative and a positive pulse of $50 \mathrm{mV}$ (to be compared with $1 \mathrm{pH}$ unit in the case of an i.s.f.e.t.) were applied to the gate, which can be seen in the $V_{0}$ curve and not in the $S_{b}$ curve, as could be expected. The shift in the time axis is due to the distance between the two pens of the double trace recorder.

It will be obvious that the $S_{b}(T)$ curve can be simply subtracted from the $V_{0}\left(T, V_{\mathrm{g}}\right)$ curve to give the desired $V_{0}\left(V_{g}\right)$ curve independent of temperature. In the meantime, the temperature signal itself is also available, which can be used in addition for a temperature-dependent amplification of $V_{0}$ in the case of an i.s.f.e.t., in accordance with the temperature dependent slope correction with normal $\mathrm{pH}$ equipment. The signal $S_{b}(T)$ can also be used for an automatic compensation if it controls $V_{r e f}$.

Summarising this Section, it was shown that a theoretically difficult to define temperature dependency of an m.o.s.f.e.t., which can also be any solidstate transducer based on the m.o.s.f.e.t. concept, can be handled with adequate electronic concepts. The quintessence of this idea is that the temperature signal is withdrawn from the measuring transducer

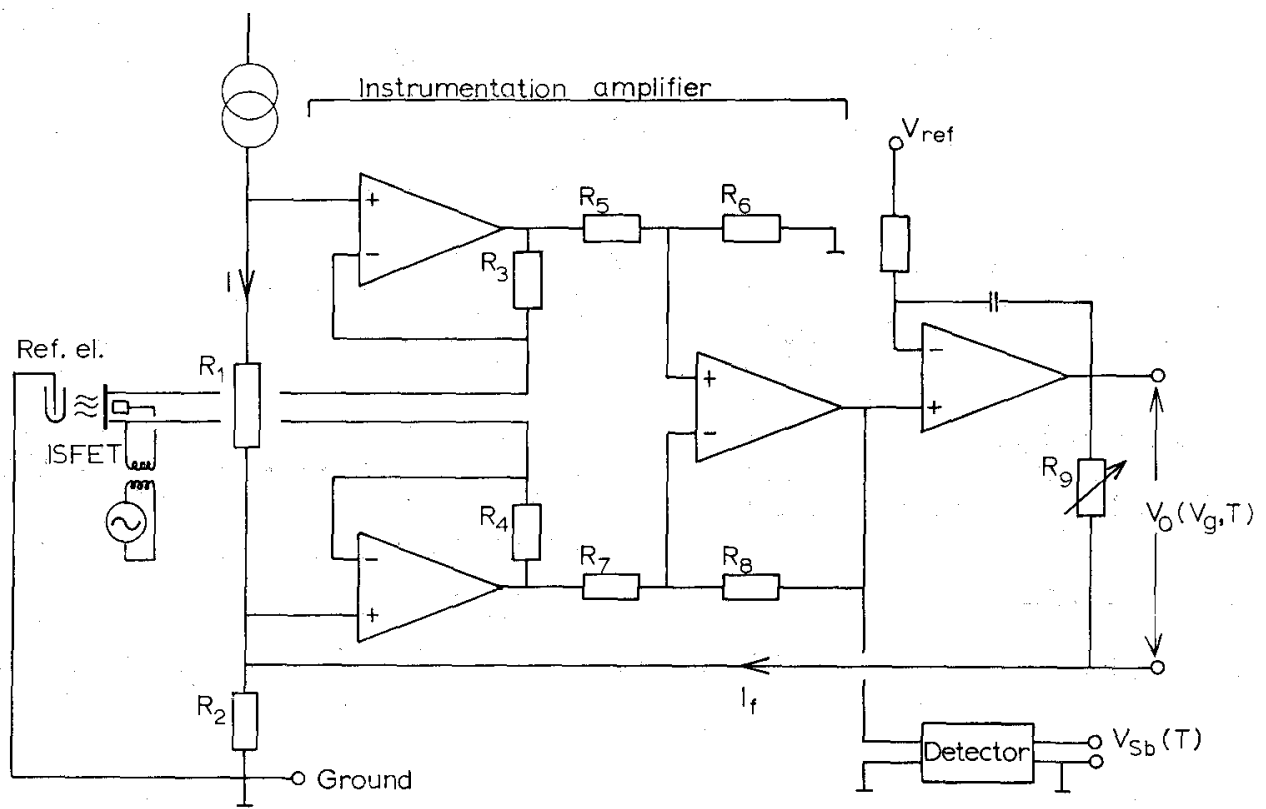

Fig. 4 Principle diagram of i.s.f.e.t. amplifier with additional circuitry for temperature measurement 
itself; a unique concept, because no problems occur with respect to temperature fluxes and thermal resistances that would certainly be the case with the

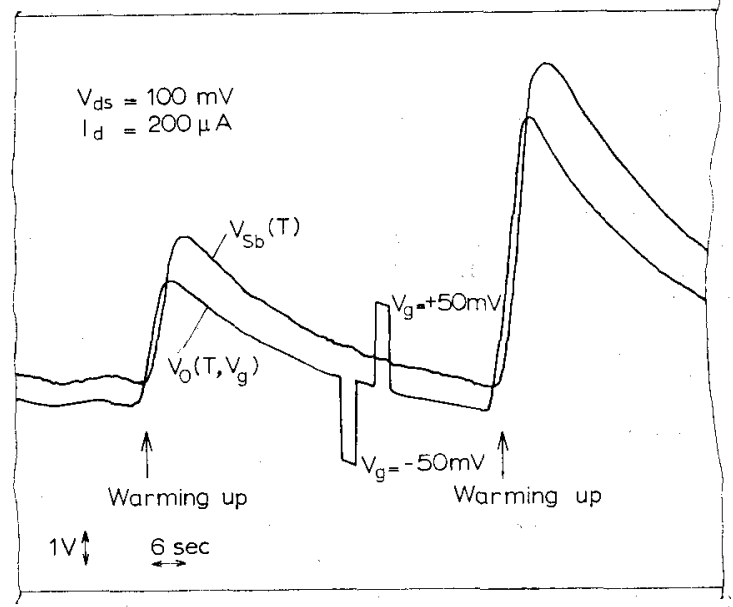

Fig. 5 Original recording of $V_{O}\left(T, V_{g}\right)$ and $V_{s b}(T)$

use of a separate sensor for the measurement of the process temperature. In the latter case it would also be a problem to match the temperature characteristics of both transducers.

\section{General conclusion and discussion}

Up to now it was argued that the advantage of the semiconductor transducers was their solid state and therefore the rigid character of the devices and the miniature design. Also the possibility of multiprobe constructions was often mentioned as a great improvement in the field of transducers. In this paper a unique concept is added to these statements, namely, a notable breakthrough with respect to the classic approach of a one-way series connection between a transducer and its corresponding electronic measuring circuit. In fact the development of semiconductor transducers is not only a modern way of constructing transducers but it introduces a whole new class of transducers that opens up tremendous possibilities in the classical field of transducers, not available up to now.

With conventional transducers, the device characteristics were fully determined by the technology of their fabrication and construction, while the new approach, described in this paper, introduces electronic concepts for controlling the transducers' device characteristics by external means. The principles described in this paper are only illustrations, but it will be obvious that similar principles applitd to other semiconductor transducers can be developed in the future.

Acknowledgment-Several students did part of the work described in this paper. The author wishes to thank especially, however, his student C. J. Stok for his great help and devotion in the discussions as well as the practical performance with respect to the contents of Section 3.

\section{References}

Bergveld, P. (1968) New amplification method for depth recording. IEEE Trans., BME-15, 102-105.

BERGVELD, P. (1969) The influence of substrate bias upon the a.c. characteristics of MOS transistors. Proc. IEEE, 57, 72-73.

BergVeld, P. (1972) Development, operation and application of the ion sensitive field effect transistor as a tool for electrophysiology. IEEE Trans., BME-19, $342-351$

Bergyeld, P., De Roois, N. F. and Zemel, J. N. (1978) Physical mechanism for chemically sensitive semiconductor devices. Nature, 273, 438-443.

Chrung, P. (Ed.) (1978) Theory, design and biomedical application of solid state chemical sensors, CRC Press, Florida, USA.

Cline, H. E. and Anthony, T. R. (1976) High-speed droplet migration in silicon. J. Appl. Phys., 47, 2325-2331.

Esashi, M. and Matsuo, T. (1978) Integrated micro multi ion sensor using field effect of semiconductor. IEEE Trans., BME-25, 184-191.

Leistiko, O., Grove, A. S. and SAH, C. T. (1965) Electron and hole mobility in inversion layers and thermally oxidized silicon surfaces. IEEE Trans., ED-12, 248-254.

Vadasz, L. and Grove, A. S. (1966) Temperature dependence of MOS transistor characteristics below saturation. IEEE Trans., ED-13, 863-866.

ZEMEL, J. N. (1977) Chemically sensitive semiconductor devices, Research/Development, 28, 38-44. 